\title{
10Gbps Vertical Cavity Surface Emitting Lasers Data Transmission
}

\author{
Winston Tumps Ireeta ${ }^{1, *}$, George Isoe ${ }^{2}$, Esther Nabadda ${ }^{1}$ \\ ${ }^{1}$ Department of Physics, Makerere University, Kampala, Uganda. \\ ${ }^{2}$ Department of Physics, Nelson Mandela University, Port Elizabeth, South Africa.
}

How to cite this paper: Winston Tumps Ireeta, George Isoe, Esther Nabadda. (2022) 10Gbps Vertical Cavity Surface Emitting Lasers Data Transmission. Engineering Advances, 2(1), 71-79. DOI: 10.26855/ea.2022.06.006

Received: February 1, 2022

Accepted: February 24, 2022

Published: March 7, 2022

*Corresponding author: Winston Tumps Ireeta, Department of Physics, Makerere University, Kampala, Uganda.

Email: winston.ireeta@mak.ac.ug

\begin{abstract}
The overwhelming increase in bandwidth demand as the number of people with smart phones and other internet-using gadgets shoots up, with emerging numerous apps (some with an aspect of live streaming), social media platforms, the increase in number of broadcasting networks both Radio and television stations, plus almost every household having a TV set and Radio, with a current advancement of access on mobile phones has created a bottle-neck in telecommunication transmissions. The blocking/denying internet access for specific apps has become the desperate measure users have resorted to, for example 'setting up a firewall for smart phones' to maintain fast internet, which is inconveniencing and user unsatisfying. Other measures in place include recent digital migration, which arose out of the Regional Radio communication Conference of 2006 (RRC06) and the subsequent Geneva 2006 Agreement (GE06) of the International Telecommunication Union (ITU) 'Recommendations' which resolved that all countries signatory to the agreement must migrate from analogue to digital broadcasting services by 2015 (Uganda was part). This is a smart move, but it doesn't solve the problem of increased required capacity. In this study, we experimentally demonstrate 10Gbps data transmissions using a direct data modulator that is, a 1300 nm Vertical Cavity Surface Emitting Laser (VCSEL). We characterized the modulator to attain effective performance thus obtaining the appropriate current value (5.5mA) at which we incurred minimum penalties and corresponding Bit Error Rates (BERs) favourable for high-speed modulation, i.e., a sensitivity of $-16.8745 \mathrm{dBm}$ power penalty was obtained on a $17 \mathrm{~km}$ length ITU-T G.652 Non-Dispersion Shifted Single mode fiber.
\end{abstract}

\section{Keywords}

Data Transmission, Vertical Cavity Surface Emitting Laser, Chromatic Dispersion, Optical Fibers

\section{Introduction}

In most areas of communication, a transition from analogue to digital communication has happened in the last few decades. The main advantage of this migration is because digital communication systems are more reliable and faster than the analogue ones. Modulation techniques used in digital and analogue communication systems differ. A bandpass signal can be got from a baseband signal using modulation processes.

An analogue signal is modulated using an analogue carrier in analogue communication systems while in digital communication systems, digital signals or binary data from the signals are taken and modulated using an analogue carrier. A modulator is the device that performs modulation while a demodulator is the device that performs demodulation.

An analogue signal is modulated by a binary code by use of mainly keying techniques in digital modulation processes. The digital modulator device is placed between the transmitter and the channel through which the signal is transmitted. 
Some of the basic types of digital modulation techniques are Amplitude Shift Keying (ASK), Frequency Shift Keying (FSK) and Phase Shift Keying (PSK) [1].

For information to be transmitted for example a baseband signal given by $X_{c}(t)$, the sinusoidal carrier signal can be represented by;

$$
\mathrm{X}_{\mathrm{c}}(\mathrm{t})=\mathrm{A}_{\mathrm{c}} \cos 2 \pi \mathrm{f}_{\mathrm{c}} \mathrm{t}+\varphi
$$

where $f_{c}$ Is carrier signal base frequency, $A_{c}$ Is the carrier signal amplitude and $\varphi$ the phase of the carrier signal.

The transmitted signal is got from a combination of the carrier signal with the baseband data signal using the modulator. Changing those parameters that is, amplitude, frequency and phase produces amplitude/intensity modulation, frequency modulation and phase modulation. Modulation can be done in two ways, direct modulation, and external modulation. Direct modulation being the easiest way especially with the availability of directly modulated VCSELs on the market.

VCSELs are attractive optical sources for emerging swept source optical coherence tomography (SS-OCT) systems and high-speed transient spectroscopy applications. SS-OCT systems targeting vascular and cancer imaging applications require greater than $100 \mathrm{~nm}$ of tuning near $1310 \mathrm{~nm}$. Prior to 2011 [2], there were no reports of integrated MEMSVCSELs in the $1310 \mathrm{~nm}$ range, and the widest reported tuning range at any wavelength was $65 \mathrm{~nm}$ at $1550 \mathrm{~nm}$. The first $1310 \mathrm{~nm}$ VCSELs with the requisite greater than $100 \mathrm{~nm}$ range for SS-OCT were demonstrated in 2011 [3]. The results presented showed record OCT imaging rates with excellent image quality. Around the same time $100 \mathrm{~nm}$ of tuning was also reported for the first time near $1550 \mathrm{~nm}$ [4] and further applications have widely been extended to optical communications.

\section{Research Design}

The experiments were carried out using: VCSEL, Power Meter (PM), Laser-diode controller (LDC), Oscilloscope (SCOPE), Pattern Generator (PPG), Mach-Zehnder, Photo Diode Detector (PIN) and Spectrum Analyzer (SA).

\section{Experiment 1 (VCSEL characterisation)}

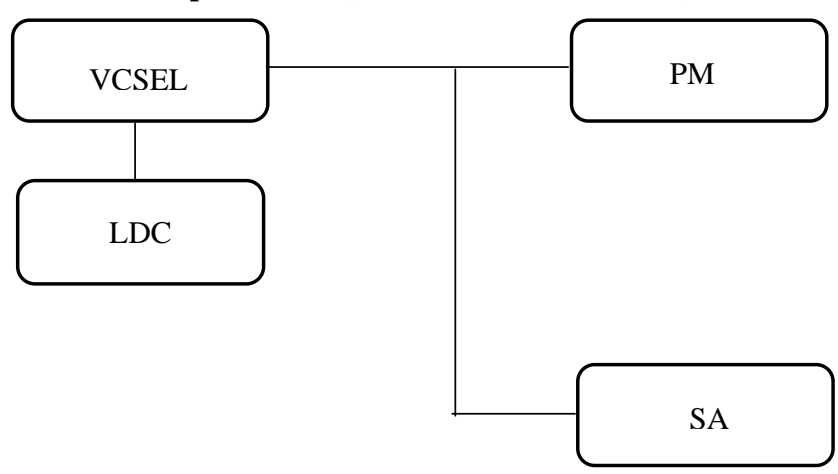

(a)

The VCSEL bias current is varied using a Laser Diode Controller to obtain the threshold, thermal roll over point and the working region. The power-meter (PM) records the corresponding change in power as the current changes.

The spectrum Analyzer demonstrates the relation of current to the wavelength at which the VCSEL transmits.

Experiment 2 (VCSEL data transmission)

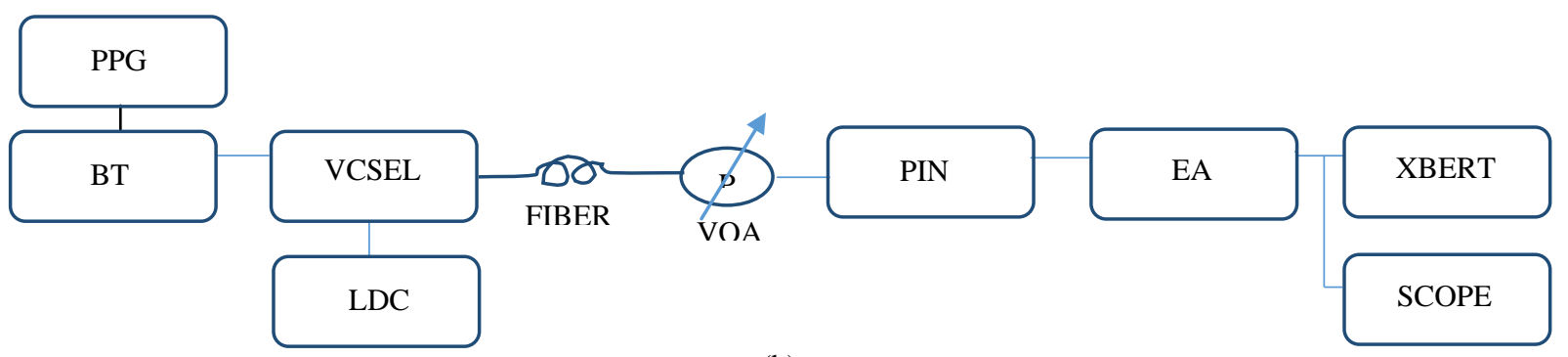

(b)

Figure 1. (a) VCSEL characterization to optimize the modulator's performance, (b) VCSEL performance at varying fibre lengths and recording the respective chromatic dispersion in the transmission link. PPG is pulse pattern generator, LDC is laser diode control, VOA is variable optical attenuator, PIN is photo diode detector, EA is electrical amplifier and BERT is bit error rate tester. 
Analysis on direct modulation is carried out, varying different parameters, i.e., current $(1310 \mathrm{~nm}$ In this study, VCSEL modulation optimization was done at different fiber lengths (by using fibers of different length) and the Chromatic dispersion effects (transmission at different windows for a G655 and G652) were determined. The reader is referred to [5] and [6] for other experimental setups that could be used.

\section{Theory}

Direct modulation is carried using a directly modulated VCSEL. The VCSEL (Vertical Cavity Surface Emitting Laser) has become the work horse of modern fiber optic communication links because of the following advantages: its ability to provide high bandwidth, single mode operation within the C-L bands, wavelength tenability, the convenience of direct modulation and energy efficiency at low drive currents. To transmit effectively, we need to obtain a region between the threshold and thermal roll over point. And this is possible using experiment 1 in Figure 1.

Considering the VCSEL structure, the gain in the cavity must balance the losses for lasing. For intrinsic losses $\alpha_{i}$ and $\alpha_{a}$ in the passive and active sections, respectively, gain enhancement and penetration of the waves into the Bragg reflectors tends to the lasing threshold condition in equation 2.

$$
\mathrm{g}_{\text {th }}=\alpha_{\mathrm{a}}+\frac{1}{\Gamma_{\mathrm{r}} \mathrm{d}_{\mathrm{a}}}\left[\alpha_{\mathrm{i}}\left(\mathrm{L}_{\mathrm{eff}}-\mathrm{d}_{\mathrm{a}}\right)+\operatorname{In} \frac{1}{\sqrt{\mathrm{R}_{\mathrm{t}} \mathrm{R}_{\mathrm{b}}}}\right]
$$

Or we can consider the photon lifetime in equation 3;

$$
\frac{1}{\tau_{p}}=\frac{d_{a}}{L_{e f f}}\left\langle V_{g r}\right\rangle \Gamma_{r} g_{t h}
$$

where; $g_{\text {th }}$ is threshold gain, $\Gamma_{r}$ the relative confinement factor or gain enhancement factor, $L_{\text {eff }}$ the effective length, $d_{a}$ total thickness of the active layer, $\mathrm{R}_{\mathrm{t}} \mathrm{R}_{\mathrm{b}}$ intensity reflection coefficient of the lossless top and the bottom mirrors respectively.

In simple terms before the threshold current is reached the power isn’t enough to run the VCSEL. In the working region, increase in the current linearly increases the driving power of the VCSEL, i.e., we expect more photons to be emitted until the point of saturation (thermal-roll over current).

Transmission at different fiber length is significant; it shows what happens to the signal as it travels longer distances. A lot of dispersions mainly (chromatic dispersion) occur and this attenuates the signal.

The received signal is analyzed using the Bit Error Receiver [7] and the Scope that gives the eye and pattern of the received digital signal.

Chromatic dispersion [8] for cases of high speeds and longer distances, a G.655 is favorable compared to the G.652.

\section{Results}

\subsection{Direct Modulation}

\section{VCSEL static characterization}

The characteristic curve explains the performance of VCSEL at increased powers and change in currents and the spectral analysis at different currents.

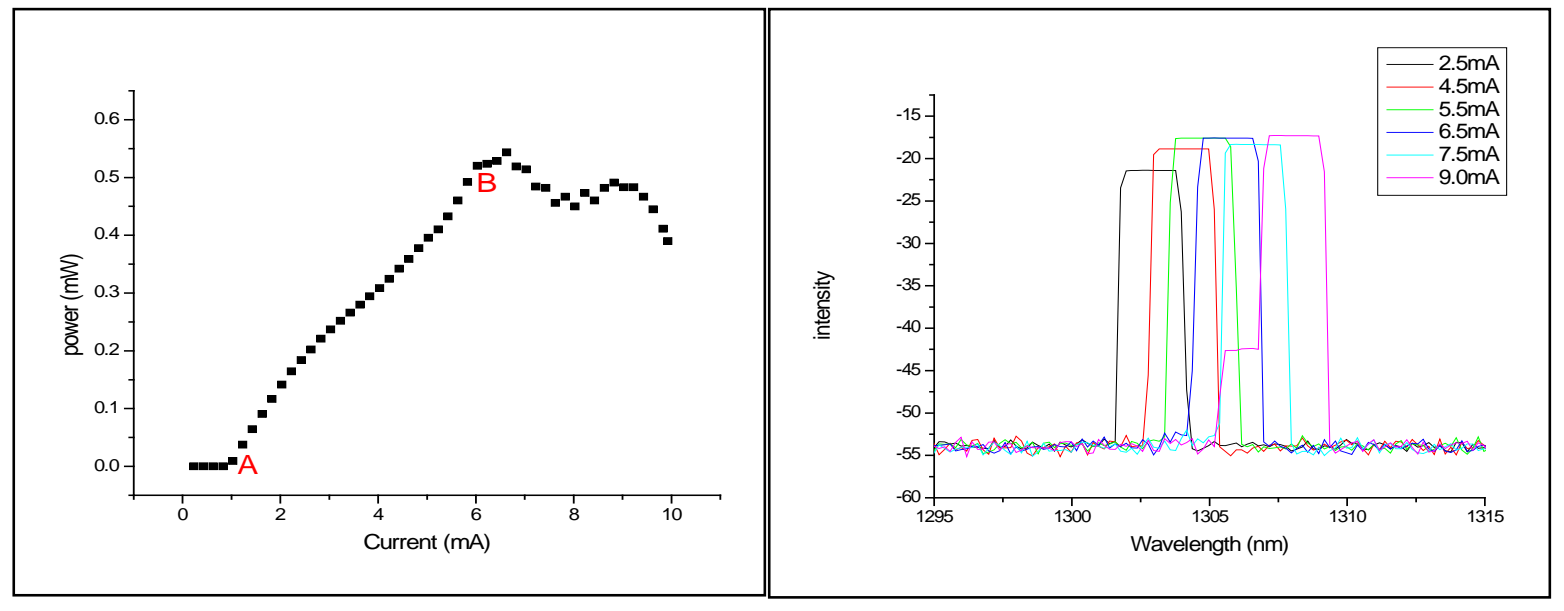

(a)

(b)

Figure 2. (a) VCSEL characteristic curve, (b) spectral analysis of a VCSEL at different bias currents. 
Before point A (threshold point) in Figure 2(a), we see no power increase even when the current ejected in the VCSEL is increasing implying no emission, between point A and B, usually referred to as the working region. In this region, increase in the current linearly increases the driving power of the VCSEL, i.e., we expect more photons to be emitted until the point of saturation (thermal-roll over current).

Above the thermal-roll over (maximum point of photon excitation), the behavior is nonlinear and unpredictable, further increase may lead to malfunctioning of the VCSEL.

According to the spectral analysis in Figure 2(b), we see an interesting behavior of the VCSEL manufactured to operate at $1310 \mathrm{~nm}$ demonstrating possibility of application within the region on 1300-1310 nm with minimum compromise in the intensity $\left(\mathrm{W} / \mathrm{m}^{2}\right)$. This shift in the wavelength indicates that transmissions can be carried out at different wavelength by tuning the VCSEL at different currents thus improved spectral efficiency of the modulator. All signals at different wavelengths (from the range of $1300 \mathrm{~nm}$ to $1310 \mathrm{~nm}$ ) show little attenuation thus provide more spectra of operation for instance in WDM systems thus the spectral efficiency.

\subsection{VCSEL modulation optimization}

The VCSEL is biased at different currents $(4.5 \mathrm{~mA}),(5.5 \mathrm{~mA}),(7.0 \mathrm{~mA})$, and $(8.5 \mathrm{~mA})$ and the Back-to-Back transmissions performed to optimize transmission as shown in Figure 3.

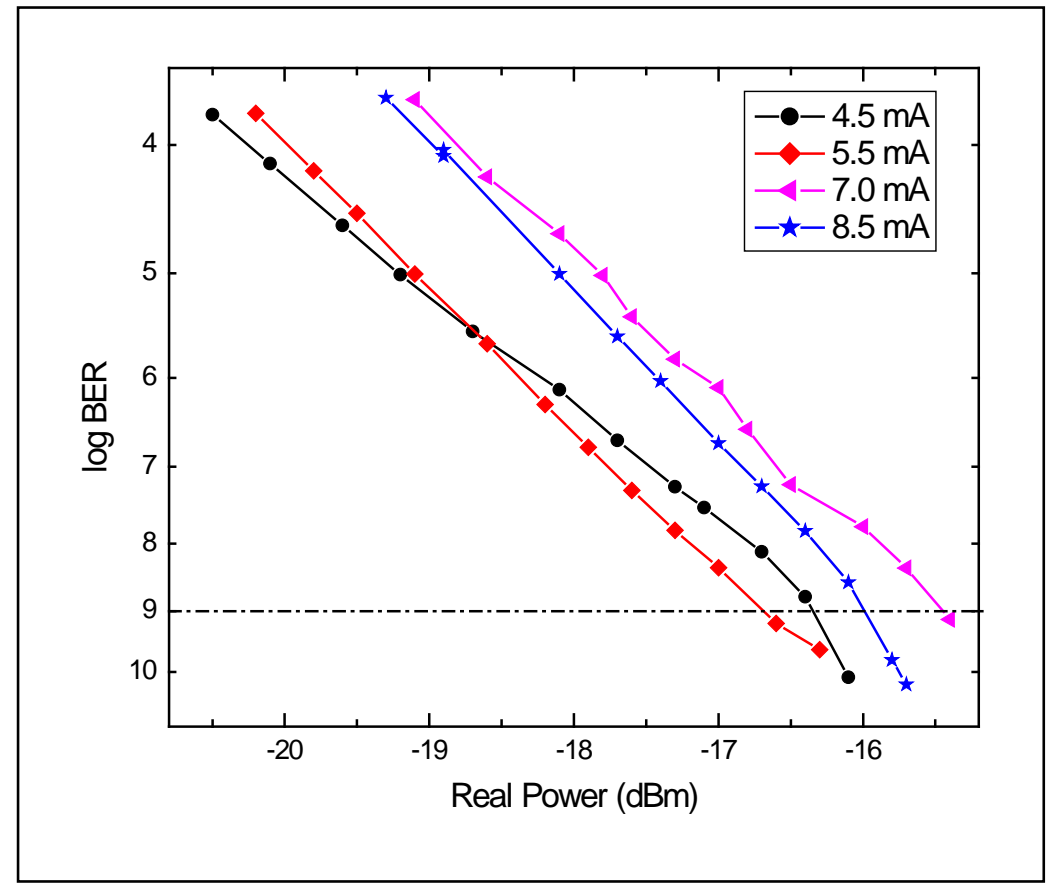

Figure 3. BER measurements as the VCSEL driving current is varied.

According to Figure 3, the bias current with the minimum penalty is $(5.50 \mathrm{~mA})$, implying this is the favorable current at which transmission should take place and the rest of our measurements were carried out at this current.

Table 1. Current versus Sensitivity of the VCSEL at different bias Currents

\begin{tabular}{ccccc}
\hline Current $(\mathrm{mA})$ & 4.5 & 5.5 & 7.0 & 8.5 \\
\hline Sensitivity $(\mathrm{dBm})$ & -16.3399 & -16.7032 & -15.9902 & -15.4832 \\
\hline
\end{tabular}

The lowest power level at which the receiver can detect a signal and demodulate data is called Receiver Sensitivity. Results of sensitivity for the VCSEL used in this study are shown in Table 1. Receiver Sensitivity is based only on receiver specification and is not dependent on the transmitter used in the digital communication system. The power density of a signal propagating from the transmitter decreases with the increase in distance which makes it difficult for the receiver to detect it. With an improvement in the receiver sensitivity (making it more negative), the radio can detect weaker signals which increases the transmission range. Receiver sensitivity is very important in the decision-making processes because a slight difference in this sensitivity can lead to large variations in the transmission range. $7.0 \mathrm{~mA}$ according to the results in Table 1 is the worst-case scenario compared to $5.5 \mathrm{~mA}$ by-1.22dBm. Let's look at the relation 
between it and 5.5mA (best scenario) using their corresponding eyes and patterns in Figure 4.

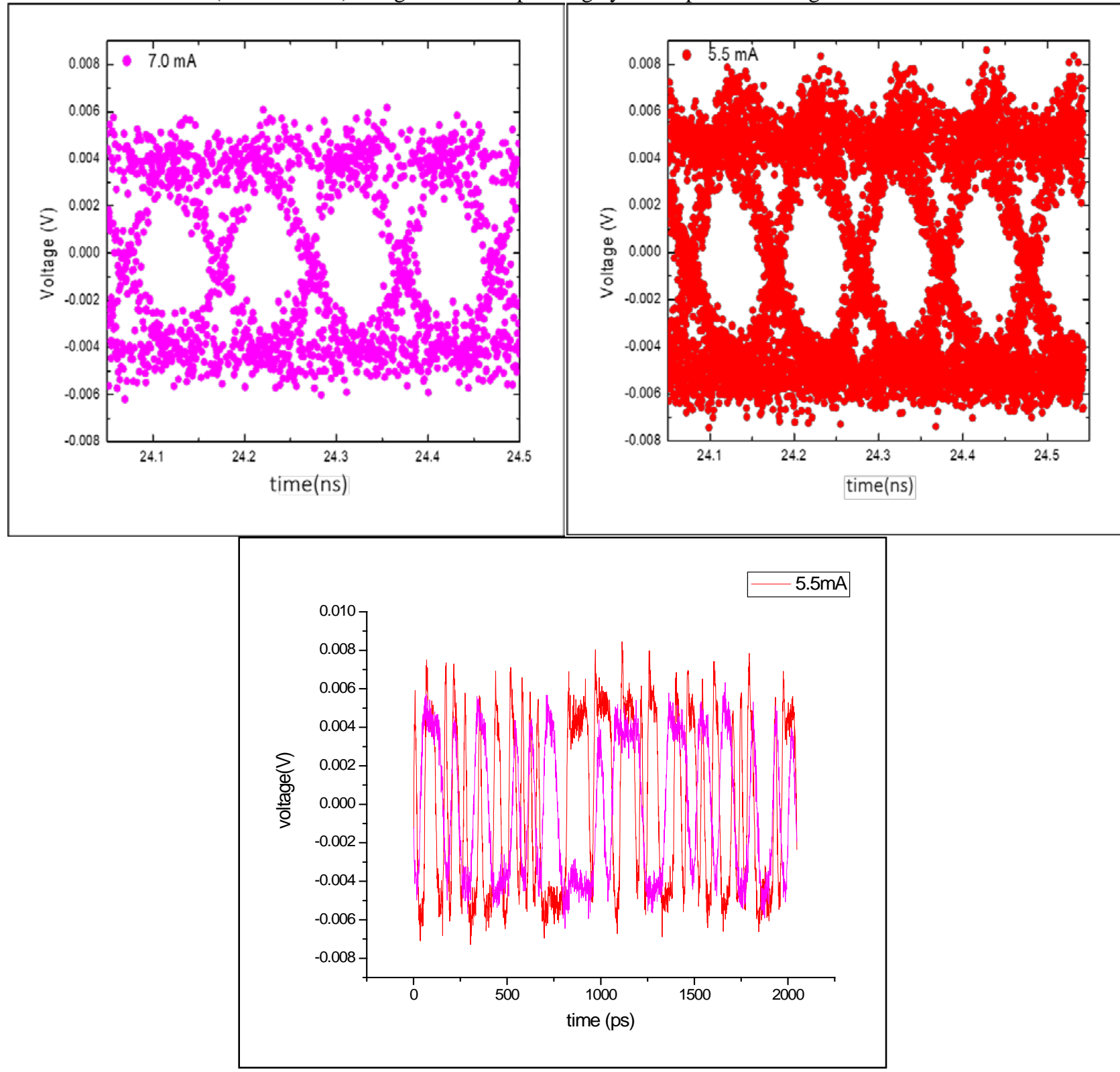

Figure 4. Eye diagrams and Patten (bits) for the worst-and best-case scenario (5.5mA driving current red and 7.0mA purple).

There is a slight closure in the eye at $7.0 \mathrm{~mA}$ and this is clearly seen on the pattern (Figure 4). A better sensitivity is because of increase in the Extinction Ratio (ER), that is, when the ratio between ' 1 ' and ' 0 ' levels increase, the chances of confusing the two on the receiver's side is minimal. And at the end of the day, the efficiency of any transmission link is based on how easy it is to accurately detect the transmitted information by the receiver. Similar results were reported in [9] and [10].

Obtaining a proper bias current, say, setting it above A preferably mid of the working region on the characteristic curve (current vs. power curve) optimizes performance. This is because, if the VCSEL bias current is very close to A or $\mathrm{B}$, the pulse width is distorted thus in turn causes jitter.

\subsection{Transmission performance of VCSEL}

Factors that affect VCSEL transmission include, lengths and chromatic dispersion; The $1310 \mathrm{~nm}$ VCSEL is modulated at a bit rate of 10 Gbs per second by a Non-Return-to- Zero (NRZ) Pseudo-Random Binary Sequence (PRBS) signal and propagated over an ITU-T G.652 Non-Dispersion Shifted single mode fiber. The length of the fiber is varied 
from $3.21 \mathrm{~km}, 11 \mathrm{~km}$ and $17 \mathrm{~km}$ as the Bit Error Rate (BER) is measured and the power penalties due to chromatic dispersion established.

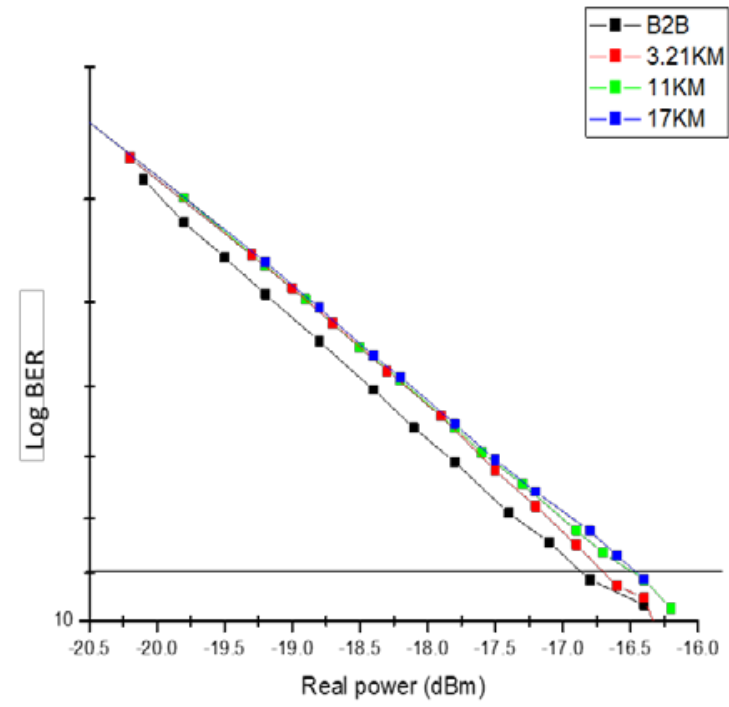

(a)

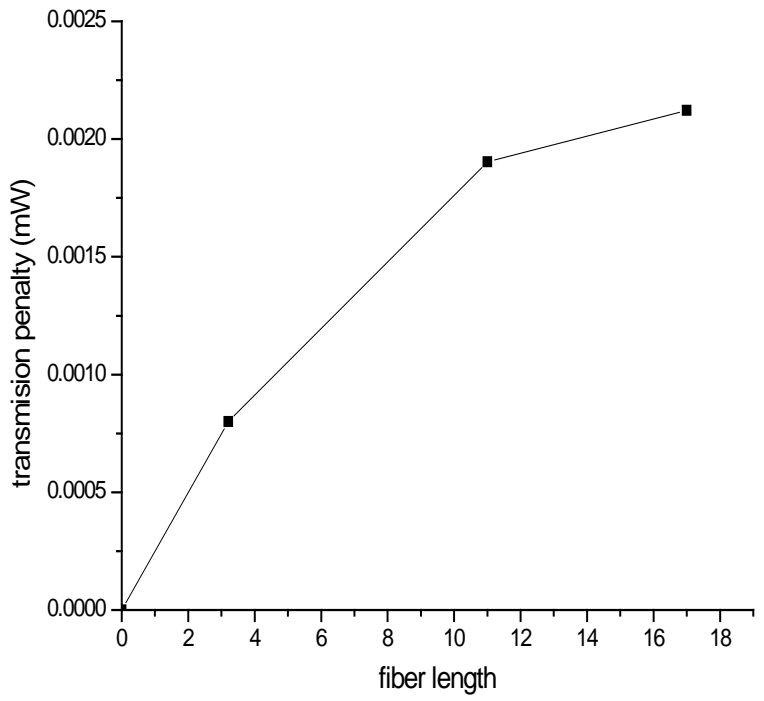

(b)

Figure 5. (a) BER measurements at different transmission length (b)Transmission penalties against distance.

The sensitivity for back-to-back transmission from figure 5 is $-16.8745 \mathrm{dBm}$.

Table 2. Sensitivities of the VCSEL at different transmission distances

\begin{tabular}{cccc}
\hline Length $(\mathrm{km})$ & 3.21 & 11 & 17 \\
\hline Penalty $(\mathrm{dBm})$ & -30.9691 & -27.2033 & -26.7325 \\
\hline
\end{tabular}

Sensitivities at $3.21 \mathrm{~km}, 11 \mathrm{~km}$ and $17 \mathrm{~km}$ are shown in Table 2.

Figures 7 and 8 show that the increase in the transmission distance increases the power penalties due to chromatic dispersion (the further the signal travels we expect more spreading), implying more power will be required to transmit at longer distances.

The closure of the eye is minimal for transmission in the $17 \mathrm{~km}$ fiber as shown in Figure 6 but using the pattern (bits), it is easily noticeable that as you increase the distance from $3.21 \mathrm{~km}$ to $17 \mathrm{~km}$ the distortions indeed increased.
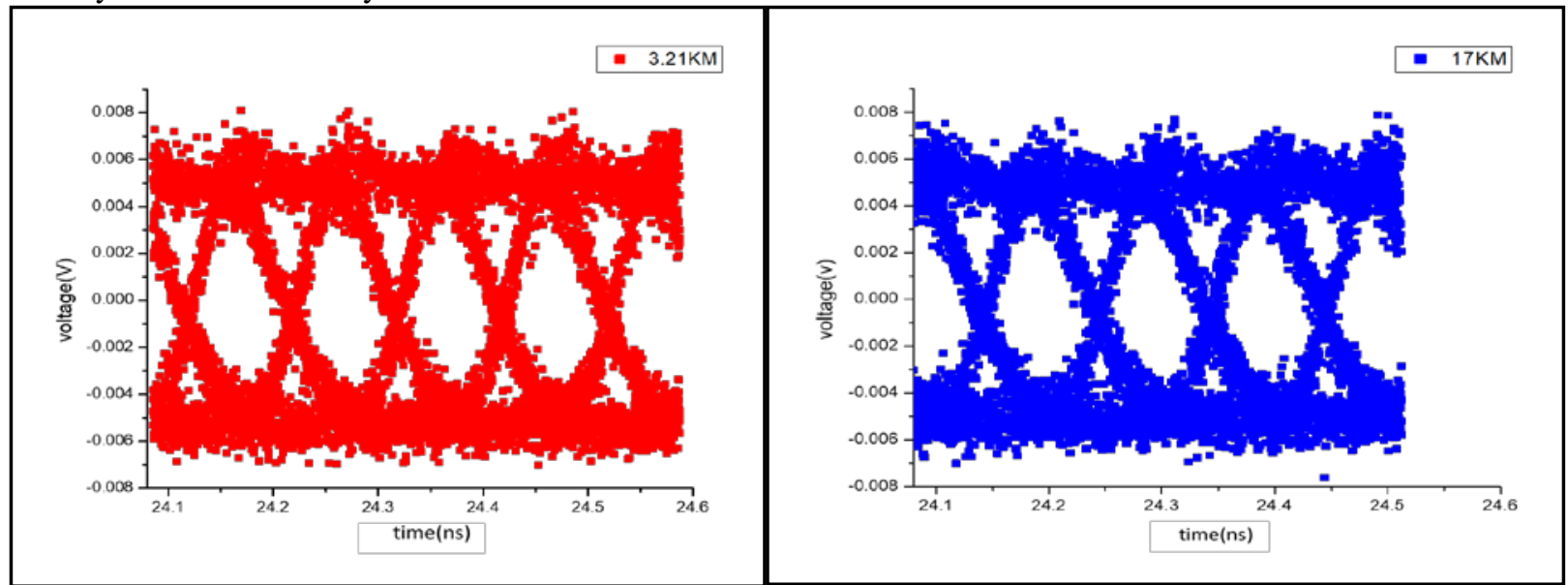


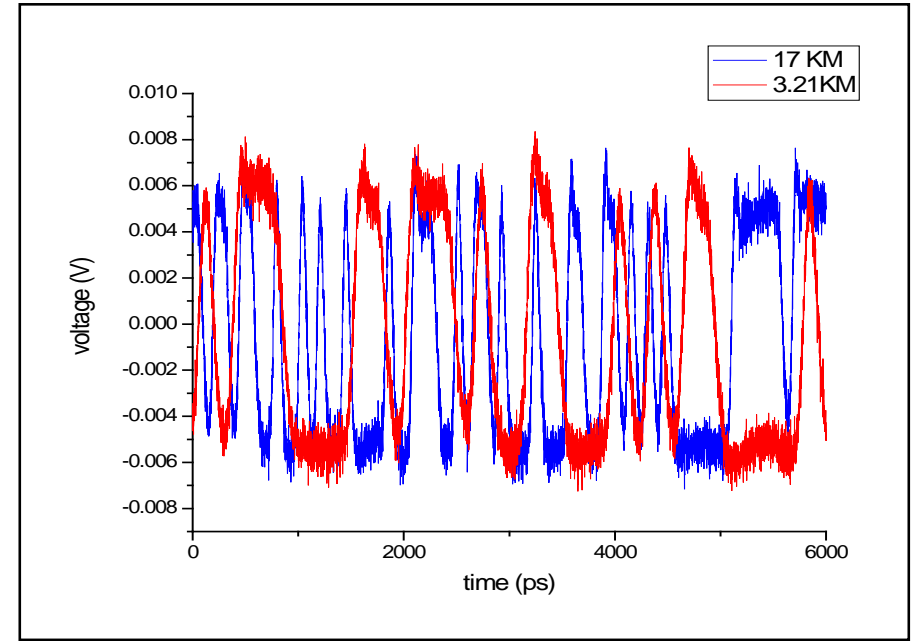

Figure 6. Transmission at $3.21 \mathrm{~km}$ and $17 \mathrm{~km}$ fibre length (best-case and worst-case scenario).

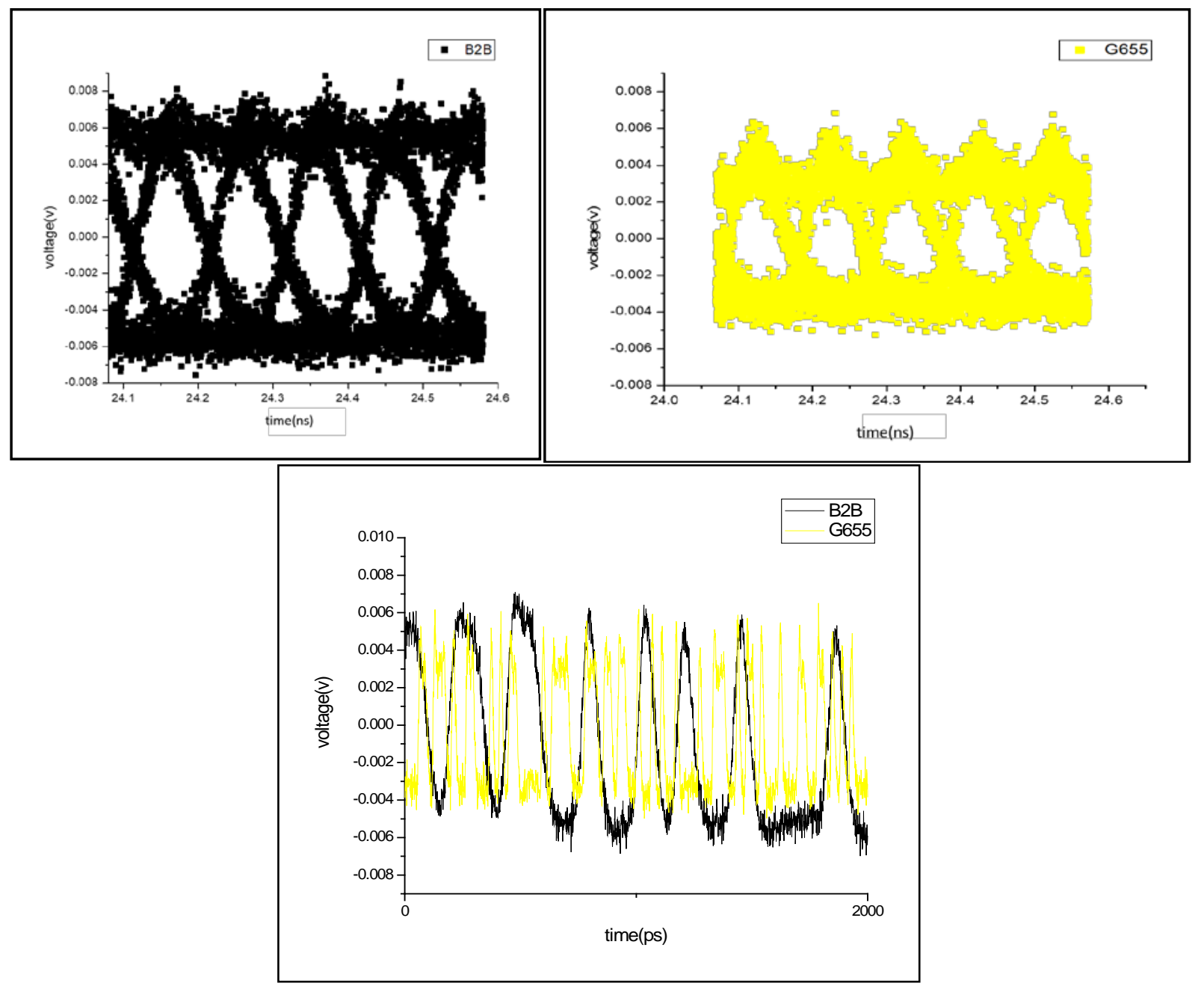

Figure 7. Corresponding Eye and Pattern for transmission at G.655 and Back-to-Back (B2B). 


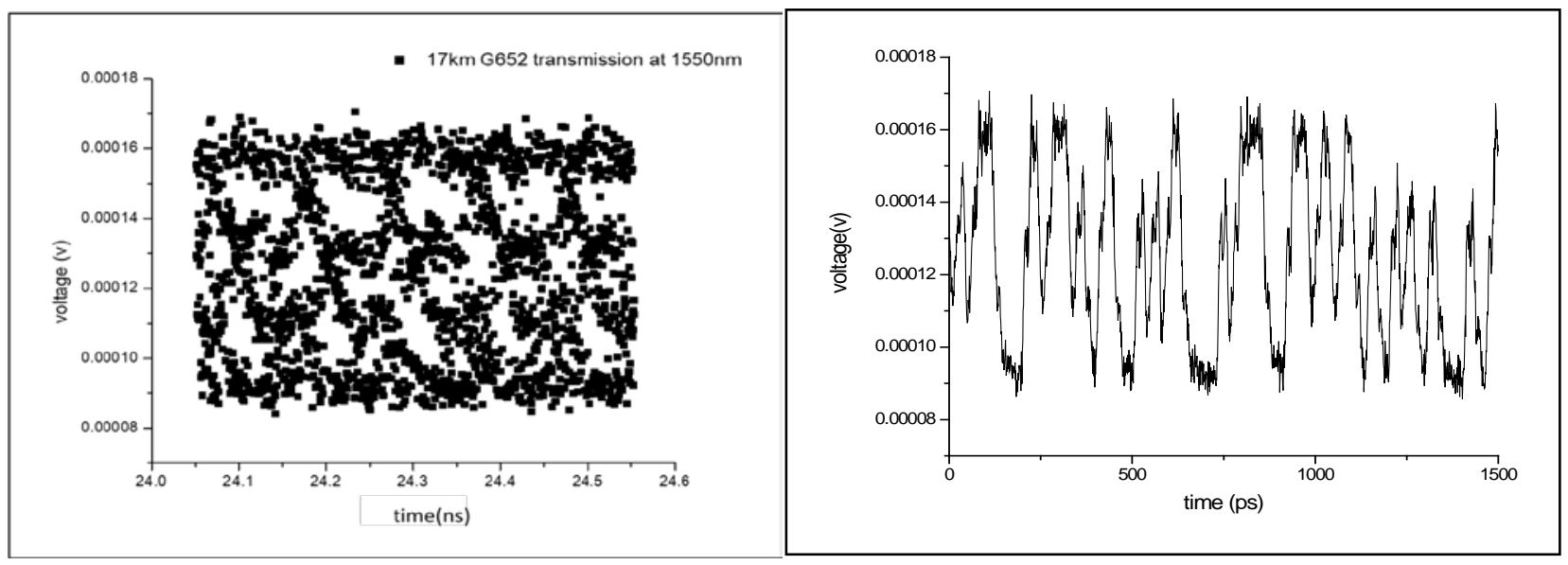

G.652 fiber transmission at 1550nm

Figure 8. Eye and pattern for a G.652 transmission at $1550 \mathrm{~nm}$.

\subsection{Chromatic dispersion effect on data transmission}

This was demonstrated by transmitting using a G.655 fiber at $1310 \mathrm{~nm}$ and G.652 at $1550 \mathrm{~nm}$ by a laser modulated at 10Gbps by a commercial Mach-Zender Modulator. The results demonstrate possibility of data transmission of G.655 with a great deal of error and inability of the G.652 fiber data transmission as in this case all the information is completely.

A G.655 is specifically manufactured to transmit with no chromatic dispersion that is why even at $1310 \mathrm{~nm}$ transmission window, not meant for its transmission, there is minimal spreading, but the penalty could not be attained as shown in Figure 7. The closure of an eye is greatly noticeable thus reduced extinction ratio and the 0 bit can easily be confused by the $1 \mathrm{bit}$, or it requires a lot of power for the receiver to differentiate between the $1 \mathrm{~s}$ and $0 \mathrm{~s}$.

A G.652 is highly permeable to chromatic dispersion and transmission at the wrong window $(1550 \mathrm{~nm})$ makes matters worse, this is evident in Figure 8. A close look at the pattern, there are points between the highest and lowest voltages that can neither be regarded as 0s or 1s thus causing distortion.

\section{Conclusion}

Using the $1310 \mathrm{~nm}$ VCSEL modulated at a bit rate of 10Gbps by a non-Return-to-zero (NRZ) Pseudo-Random Binary Sequence (PRBS) signal propagated over an ITU-T G.652 Non-Dispersion Shifted Single Mode fiber of length 17 $\mathrm{km}$, a sensitivity of $-16.8745 \mathrm{dBm}$ power penalty was achieved.

\section{Conflict of Interest}

On behalf of all authors, the corresponding author states that there is no conflict of interest.

\section{References}

[1] G. Tharakanatha, S. K. Mahaboob kamal basha, Vijay Bhaskar chanda, I. Hemalatha. (2013). "Implementation and Bit Error Rate analysis of BPSK Modulation and Demodulation Technique using MATLAB”, International Journal of Engineering Trends and Technology (IJETT), Vol. 4(9), pp. 4010-4014, Sep 2013.

[2] Iiyama, K., S. Matsui, and T. Kobayashi. (2011). "High-resolution FMCW reflectometry using a single-mode vertical-cavity surface-emitting laser.” Photonics Technology Letters. 2011.

[3] Jayaraman. (2011). “OCT imaging up to 760KHz axial scan rate using single mode 1310nm MEMS-tunable VCSELs.” Laser Science to Photonic Application. 2011.

[4] Zogal, Karolina, Tobias Gruendl, Hooman A. Davani, Christian Gierl, Sandro Jatta, Christian Grasse, Markus C. Amann, and Peter Meissner. (2011). “High Speed Modulation of a 1.55- $\mu \mathrm{m}$ MEMS-tunable VCSEL.” osapublishing. 2011.

[5] Kanakis, G., Iliadis, N., Soenen, W., Moeneclaey, B., Argyris, N., Kalavrouziotis, D., Spiga, S., Bakopoulos, P., Avramopoulos, H. (2019). High-Speed VCSEL-Based Transceiver for 200 GbE Short-Reach Intra-Datacenter Optical Interconnects. Appl. Sci. 2019, 9, 2488. https://doi.org/10.3390/app9122488.

[6] H. Kao, C. Tsai, S. Leong, C. Peng, Y. Chi, J. Huang, H. Kuo, T. Shih, J. Jou, W. Cheng, C. Wu, and G. Lin. (2017). “Com- 
parison of single-/few-/multi-mode 850 nm VCSELs for optical OFDM transmission,” Opt. Express, 25, 16347-16363 (2017).

[7] P. K. Sun and G. Lowe. (1993). "XBERT - A versatile $622 \mathrm{Mb} / \mathrm{sec}$ bit error rate generator/receiver methodology for SONET OC-3 application,” Sixth Annual IEEE International ASIC Conference and Exhibit, 1993, pp. 358-361, doi: 10.1109/ASIC.1993.410738.

[8] E. K. Rotich Kipnoo, H. Y. S. Kourouma, R. R. G. Gamatham, A. W. R. Leitch, and T. B. Gibbon. (2016). "Chromatic Dispersion Compensation for VCSEL Transmission for Applications such as Square Kilometre Array South Africa,” South African Institute of Physics, 2016.

[9] Chuan Xie, Neinyi Li, Shenghong Huang, Chiyu Liu, Li Wang, Kenneth P. Jackson. (2013). "The next generation high data rate VCSEL development at SEDU,” Proc. SPIE 8639, Vertical-Cavity Surface-Emitting Lasers XVII, 863903 (13 March 2013); https://doi.org/10.1117/12.2008815.

[10] L. Giovane, J. Wang, M. Murty, A. Harren, H. Chang, C. Wang, D. Hui, Z. Feng, T. Fanning, A. Sridhara, S. Taslim, and J. Chu. (2016). "Volume Manufacturable High speed 850nm VCSEL for 100G Ethernet and Beyond,” in Optical Fiber Communication Conference, OSA Technical Digest (online) (Optical Society of America, 2016), paper Tu3D.5. 